\title{
Validation of the American Joint Committee on Cancer (AJCC) 8th edition stage system for gastric cancer patients: a population-based analysis
}

\author{
Xingkang $\mathrm{He}^{1,2} \cdot$ Wenrui $\mathrm{Wu}^{3,4} \cdot$ Zhenghua $\mathrm{Lin}^{2,5} \cdot$ Yue Ding ${ }^{1,2} \cdot$ Jianmin $\mathrm{Si}^{1,2}$. \\ Lei-min Sun ${ }^{1,2}$
}

Received: 11 July 2017 / Accepted: 23 September 2017 / Published online: 20 October 2017

(C) The International Gastric Cancer Association and The Japanese Gastric Cancer Association 2017

\begin{abstract}
Background Our aim was to validate the American Joint Committee on Cancer (AJCC) 8th edition stage system for gastric cancer in the Western world and to compare several modifications between the 7 th and 8 th edition systems.

Methods Eligible patients having undergone surgical resection of gastric cancer during 2004-2011 from the Surveillance, Epidemiology, and End Results (SEER) database were included in the current study. Survival differences were assessed by Kaplan-Meier curve and log-rank tests. The discriminative power of the AJCC 8th and 7th editions was compared by Harrell's concordance index (c-index).

Results Patients with $\mathrm{pN} 3 \mathrm{a}$ and $\mathrm{pN} 3 \mathrm{~b}$ presented distinct survival outcomes, especially for cases in which more than
\end{abstract}

Electronic supplementary material The online version of this article (doi:10.1007/s10120-017-0770-1) contains supplementary material, which is available to authorized users.

\section{Jianmin Si}

Jianmin_si@zju.edu.cn

Lei-min Sun

sunlm@zju.edu.cn

1 Department of Gastroenterology, Sir Run Run Shaw Hospital, Zhejiang University Medical School, Hangzhou 310016, Zhejiang, China

2 Institute of Gastroenterology, Zhejiang University (IGZJU), Hangzhou, China

3 State Key Laboratory for Diagnosis and Treatment of Infectious Diseases, The First Affiliated Hospital, School of Medicine, Zhejiang University, Hangzhou, China

4 Collaborative Innovation Center for Diagnosis and Treatment of Infectious Diseases, Hangzhou, China

5 Department of Gastroenterology, The Second Affiliated Hospital of Zhejiang University Medical College, Hangzhou, China
15 lymph nodes were examined. The overall (OS) and cancer-specific survival (CSS) c-indices for the 8th edition were largely comparable with c-indices for the 7 th edition throughout the cohort. Notably, the new edition improved the power of discrimination slightly in OS and CSS (c-indices: $0.717,0.744$ ) compared with the 7 th edition (c-indices: $0.712,0.739$ ) for patients for whom 15 or more lymph nodes were examined. The analysis of stage migration in the new edition revealed nonhomogeneous survival outcomes in stages IIIB and IIIC.

Conclusion The AJCC 8th stage system for gastric cancer performs as well as the AJCC 7th edition in the United States (USA). Importantly, when more than 15 lymph nodes are examined, the discriminatory performance of the new edition is improved.

Keywords Gastric cancer - AJCC 8th stage system SEER
Abbreviations
AJCC American Joint Committee on Cancer
OS Overall survival
CSS Cancer-specific survival
SEER Surveillance, Epidemiology, and End Results
TNM Tumour, lymph node, metastasis
ICD-O-3 International Classification of Disease for Oncology, 3rd edition
IGCA The International Gastric Cancer Association

\section{Introduction}

Despite the declining incidence trend over the past decades, gastric cancer accounts for a great many cancer-related deaths and remains a considerable health burden globally 
[1-3]. The prognosis of early gastric cancer after radical surgery is favorable $[4,5]$. However, survival outcomes for advanced gastric cancer are dismal [6]. An accurate and uniform staging system for cancer is essential for physicians to tailor appropriate therapeutic decisions, as well as to inform patients regarding their prognosis, and to evaluate different treatment options, thus facilitating continuing investigation [7-9].

Currently, the tumour-lymph node-metastasis (TNM) stage system based on the anatomic extent of malignancies remains the benchmark in clinical practice globally. The first edition of the TNM stage system was released by the Union for International Cancer Control (UICC) and American Joint Committee on Cancer (AJCC) in 1977 [10]. During the past 40 years, it has undergone several major revisions, and the current AJCC 7th edition for gastric cancer was published in 2004 [11]. Several important modifications (refinements of $\mathrm{pT}, \mathrm{pN}$, stage categories) were introduced into the 7 th edition [12], and its validity has been demonstrated by previous studies [13-15]. However, the pN3b category was not incorporated into any stage subgroups in the 7th edition, and additional stage subgroups (stages IIB and IIIC) failed to improve predictive ability in stage-based prognosis [16].

In view of these shortcomings, the UICC/AJCC published the 8th edition stage system last year, and this new system will be implemented in 2018. Several small modifications have been incorporated into the new pathological stage classification, including inclusion of $\mathrm{pN} 3 \mathrm{~b}$ into the stage system and modifications of existing stage groupings [17]. These evidence-based revisions were based on the International Gastric Cancer Association (IGCA) staging project [16]. Patients in the IGCA project were mainly Japanese and Korean (84.8\%), and Western cases accounted for only $8.8 \%$. As is known, the incidence and prognosis of gastric cancer present with significant geographic differences [18, 19], which may be subject to selection bias and inevitably raise the concern whether the 8th edition system is appropriate and suitable for Western populations. Therefore, we conducted a population-based study to assess the prognostic performance of this new edition in the USA. In addition, we also wanted to examine whether the changes of the 8th edition resulted in improved discriminatory ability as compared to the 7 th edition.

\section{Methods}

\section{Study cohort}

Data were obtained from the National Cancer Institute's surveillance, epidemiology, and end results (http://seer.cancer. gov/) by SEER*Stat Software. We identified gastric cancer patients using the following inclusion criteria: patients with primary microscopically confirmed stomach cancer (primary site code, C16.1 through C16.9, exclusion of cardiac localization), aged $\geq 18$ years, histology codes of adenocarcinoma (the International Classification of Disease for Oncology 3rd edition: M-8140/3, M-8142/3 to M-8145/3, M-8210/3, M-8211/3, M-8255/3, M-8260/3 to M-8263/3, M-8310/3, M-8323/3, M-8480/3, M-8481/3, M-8490/3), who underwent surgical resection (Surg Prim Site code, 30-80). The pT, $\mathrm{pN}$, and $\mathrm{pM}$ categories of patients were redefined using information available in SEER about collaborative stage extension (2004+), Collaborative Stage (CS) regional nodes positive $(1988+)$, and CS metastases (mets) at distant sites (dx) (2004+), respectively. Stage subgroups were determined according to the 7th edition and 8th edition staging manuals. We included patients who were diagnosed between 2004 and 2011 to ensure at least 5 years of followup. The current study is considered exempt by the institutional review board of Sir Run Run Shaw hospital because all data are public.

\section{Statistical analysis}

Overall survival (OS) and cancer-specific survival (CSS) were compared by Kaplan-Meier estimates and examined by log-rank tests. Cox proportional hazards models were adopted to determine the risk factors of OS and CSS. Such variables as age, sex, year, race, marital status, tumour grade, radiation, surgery, chemotherapy, and number of lymph nodes examined were adjusted. The Harrell's c-index (c-index) [20] was used to compare the relative discriminative abilities of the two staging systems. The c-index measures the general predictive power of a regression mode [21], and a value close to 1 signifies a perfect predictive ability. All analyses were two sided, and a value of $P<0.05$ was considered statistically significant. All analyses were carried out with R version 3.4 (http://www.r-project.org) and STATA version 13.0 (Stata Corp, College Station, TX, USA).

\section{Results}

From 2004 to 2011, a total of 10,451 patients having undergone surgical resection of gastric cancer fulfilled the inclusion criteria. The median follow-up period of the included cohort was 8.7 years. Detailed characteristics of cases are summarized in Table 1. More than half the patients were male, married, and aged $>65$ years. One third of cases were located at the gastric antrum, and approximately two thirds of the tumors were poorly differentiated or undifferentiated. In the 8th edition system, most cases were classified into pT3 and pT4 (67.44\%). Almost two thirds of patients had lymph node metastases, and at least 15 lymph nodes were 
Table 1 Clinicopathological features of patients with gastric cancer

\begin{tabular}{lll}
\hline Characteristic & Num- & Percentage (\%) \\
& ber of \\
& patients & \\
\hline
\end{tabular}

Age (years)

$\leq 65$

$>65$

Gender

Male

Female

Marital status

Married

Unmarried

Unknown

Race

Non-Hispanic White

Non-Hispanic Black

Hispanic (all races)

Non-Hispanic American Indian/Alaska

Non-Hispanic Asian or Pacific Islander 2509

Non-Hispanic unknown race

Years

2004-2007

2008-2011

Grade

G1/G2

$\mathrm{G} 3 / \mathrm{G} 4$

Unknown

Location

Fundus of stomach $\quad 411$

Body of stomach $\quad 1229 \quad 11.76$

Gastric antrum $\quad 3726 \quad 35.65$

Pylorus

Lesser curvature of stomach, NOS

$629 \quad 6.02$

$1598 \quad 15.29$

$676 \quad 6.47$

Greater curvature of stomach, NOS

Overlapping lesion of stomach

Stomach, NOS

1049

1133

10.04

Lymph nodes examined

$<15$

$\geq 15$

5383

4996

51.51

Unknown

72

47.80

AJCC 8th pT category

T1

$2197 \quad 21.02$

$\mathrm{T} 2$

T3

T4a

21.02

3508

11.54

2485

33.57

$\mathrm{T} 4 \mathrm{~b}$

1055

23.78

AJCC 8th pN category

\begin{tabular}{lll} 
N0 & 3942 & 37.72 \\
N1 & 1838 & 17.59 \\
N2 & 1883 & 18.02 \\
N3a & 1882 & 18.01 \\
\hline
\end{tabular}

Table 1 (continued)

\begin{tabular}{|c|c|c|}
\hline Characteristic & $\begin{array}{l}\text { Num- } \\
\text { ber of } \\
\text { patients }\end{array}$ & Percentage $(\%)$ \\
\hline $\mathrm{N} 3 \mathrm{~b}$ & 906 & 8.67 \\
\hline \multicolumn{3}{|l|}{ AJCC 8th pM categories } \\
\hline M0 & 9121 & 87.27 \\
\hline M1 & 1330 & 12.73 \\
\hline \multicolumn{3}{|l|}{ AJCC 8th pStage } \\
\hline IA & 1778 & 17.01 \\
\hline IB & 893 & 8.54 \\
\hline IIA & 1292 & 12.36 \\
\hline IIB & 1149 & 10.99 \\
\hline IIIA & 1768 & 16.92 \\
\hline IIIB & 1457 & 13.94 \\
\hline IIIC & 784 & 7.50 \\
\hline IV & 1330 & 12.73 \\
\hline \multicolumn{3}{|l|}{ Surgery $^{\mathrm{a}}$} \\
\hline Nontotal gastrectomy & 7759 & 74.24 \\
\hline Total gastrectomy & 2692 & 25.76 \\
\hline \multicolumn{3}{|l|}{ Radiation } \\
\hline Radiation & 3073 & 29.40 \\
\hline No/unknown ${ }^{\mathrm{b}}$ & 7240 & 69.28 \\
\hline Unknown & 138 & 1.32 \\
\hline \multicolumn{3}{|l|}{ Chemotherapy } \\
\hline Chemotherapy & 4617 & 44.18 \\
\hline No/unknown ${ }^{\text {b }}$ & 5834 & 55.82 \\
\hline
\end{tabular}

AJCC American Joint Committee on Cancer, G1 well differentiated, $G 2$ moderately differentiated, $G 3$ poorly differentiated, $G 4$ undifferentiated

a Total gastrectomy was defined as codes 40, 41, 42, 52, 60, 62, and 63 from Surg Prim Site code in SEER and nontotal gastrectomy was defined as any other codes

${ }^{b}$ No/unknown indicates that no evidence of chemotherapy or radiation was found in the medical records examined

examined in fewer than half the cases. In this study, stage III was the most common stage group.

In the 8th edition, both overall survival rate and cancerspecific survival rate decreased in a stepwise fashion with increasing $\mathrm{pT}$ and $\mathrm{pN}$ categories (Fig. 1). Multivariable Cox analysis also demonstrated that high risks of overall and cancer-specific death were associated with increasing $\mathrm{pT}$ and $\mathrm{pN}$ categories even after adjusting for other variables (Table 2), suggesting the well-established predication of $\mathrm{pT}$ and $\mathrm{pN}$ in OS and CSS. The principal modification of the AJCC 8th edition is the separation of the $\mathrm{pN} 3 \mathrm{~b}$ from the $\mathrm{pN} 3$ category and the incorporation of $\mathrm{pN} 3 \mathrm{~b}$ into IIIB/IIIC stage groups. Significant OS and CSS differences between $\mathrm{pN} 3 \mathrm{a}$ and $\mathrm{pN} 3 \mathrm{~b}$ were also noted in current studies (Supplemental Fig. 1; logrank test, $P<0.01$ ). It is commonly acknowledged that the number of lymph nodes examined could affect the accuracy 
a

Kaplan-Meier survival estimates

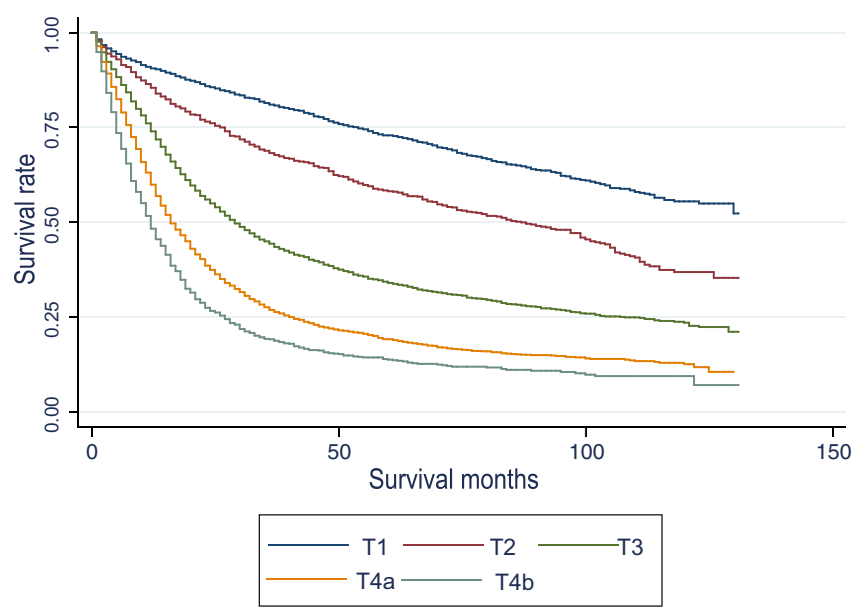

C

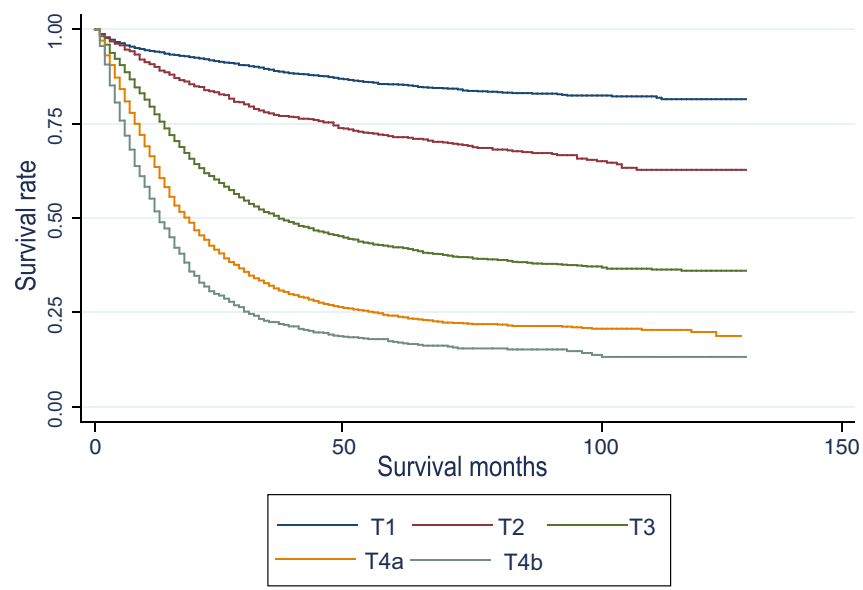

Fig. 1 Kaplan-Meier survival curves of overall and cancer-specific survival of gastric cancer according to $\mathrm{pT}$ and $\mathrm{pN}$ categories in the American Joint Committee on Cancer (AJCC) 8th edition. a Overall

of $\mathrm{pN}$ staging [22]. Thus, we further examined survival differences in patients for whom more than 15 lymph nodes were examined. In OS and CSS Kaplan-Meier curves, the distance between curves became wider, reflecting the better stratification of mortality risks in patients with more than 15 lymph nodes examined. To specifically evaluate the prognostic utility of pN3a and pN3b, subgroup analyses stratified by pT category were conducted. As the number of patients was limited $(N=38)$ in the pT1 group, no significant difference between $\mathrm{pN} 3 \mathrm{a}$ and $\mathrm{pN} 3 \mathrm{~b}$ was found. In patients with $\mathrm{pT} 3 /$ pT4a tumours, pN3b nodal status was associated with worse OS and CSS (all $P<0.01$ ). However, survival difference between pN3a and pN3b in pT2 and pT4b groups became nonsignificant (all $P>0.05$; Fig. 2, Supplemental Fig. 2). Survival differences between $\mathrm{pN} 3 \mathrm{a}$ and $\mathrm{pN} 3 \mathrm{~b}$ appeared to be influenced by the pT category. Surprisingly, for cases for b

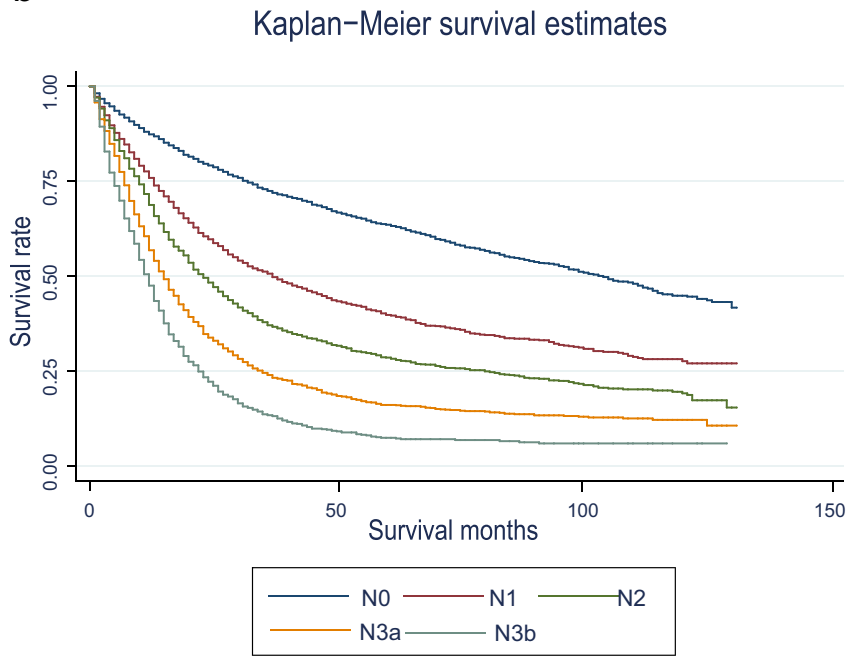

d

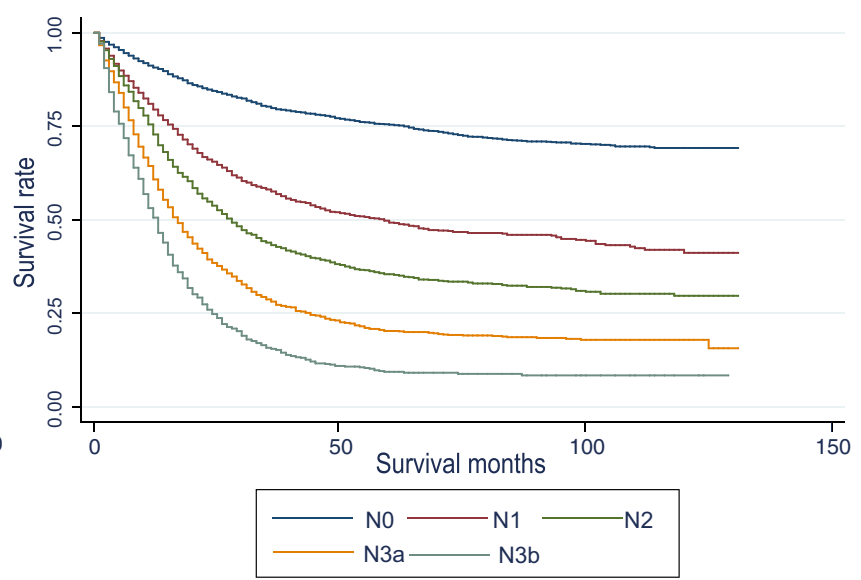

survival stratified by pT category. b Overall survival stratified by $\mathrm{pN}$ category. c Cancer-specific survival stratified by pT category. d Cancer-specific survival stratified by $\mathrm{pN}$ category

which more than 15 lymph nodes were examined, significant differences between $\mathrm{pN} 3 \mathrm{a}$ and $\mathrm{pN} 3 \mathrm{~b}$ were also observed, even in the pT2 and pT4b subgroups (data not shown). This result suggested to us that a recommendation of a minimum of 16 examined lymph nodes could improve the prognostication role of $\mathrm{pN} 3 \mathrm{a}$ and $\mathrm{pN} 3 \mathrm{~b}$.

Subsequently, we calculated the short-term (1-year) and long-term (5-year) stage-related survival rates of the two edition systems. As Fig. 3 shows, both the 7th and 8th editions provided good stratification of OS and CSS. Comparing stages IIIA, IIIB, and IIIC in the ASCC 7th edition with the ASCC 8th edition, patients with the same stages had worse OS and CSS. Multivariable Cox analysis also determined the prognostic role of the AJCC 8th stage system. A more advanced stage indicated markedly higher risks of overall and cancer-specific death (Table 2) (stage IA as 
Table 2 Multivariate Cox analysis of $\mathrm{T}, \mathrm{N}$, and stage classification on overall and cancer-specific survival in AJCC 8th edition staging system

\begin{tabular}{|c|c|c|c|c|c|c|}
\hline \multirow[t]{2}{*}{ AJCC 8th edition } & \multicolumn{3}{|c|}{ Overall survival } & \multicolumn{3}{|c|}{ Cancer-specific survival } \\
\hline & $\mathrm{HR}^{\mathrm{a}}$ & $(95 \% \mathrm{CI})$ & $P$ & $\mathrm{HR}^{\mathrm{a}}$ & $(95 \% \mathrm{CI})$ & $P$ \\
\hline \multicolumn{7}{|l|}{$\mathrm{T}$} \\
\hline $\mathrm{T} 1$ & Reference & & & Reference & & \\
\hline $\mathrm{T} 2$ & 1.81 & $(1.62,2.02)$ & $<0.001$ & 2.24 & $(1.93,2.60)$ & $<0.001$ \\
\hline $\mathrm{T} 3$ & 3.61 & $(3.31,3.93)$ & $<0.001$ & 5.86 & $(5.20,6.60)$ & $<0.001$ \\
\hline $\mathrm{T} 4 \mathrm{a}$ & 5.74 & $(5.24,6.28)$ & $<0.001$ & 9.69 & $(8.58,10.94)$ & $<0.001$ \\
\hline $\mathrm{T} 4 \mathrm{~b}$ & 7.45 & $(6.72,8.25)$ & $<0.001$ & 12.80 & $(11.22,14.60)$ & $<0.001$ \\
\hline \multicolumn{7}{|l|}{$\mathrm{N}$} \\
\hline No & Reference & & & Reference & & \\
\hline N1 & 2.41 & $(2.24,2.61)$ & $<0.001$ & 2.98 & $(2.72,3.26)$ & $<0.001$ \\
\hline $\mathrm{N} 2$ & 3.48 & $(3.23,3.76)$ & $<0.001$ & 4.44 & $(4.06,4.86)$ & $<0.001$ \\
\hline $\mathrm{N} 3 \mathrm{a}$ & 5.58 & $(5.17,6.03)$ & $<0.001$ & 7.32 & $(6.69,8.02)$ & $<0.001$ \\
\hline $\mathrm{N} 3 \mathrm{~b}$ & 8.29 & $(7.51,9.16)$ & $<0.001$ & 11.29 & $(10.10,12.62)$ & $<0.001$ \\
\hline \multicolumn{7}{|l|}{ Stage } \\
\hline IA & Reference & & & Reference & & \\
\hline IB & 1.63 & $(1.43,1.86)$ & $<0.001$ & 2.01 & $(1.65,2.45)$ & $<0.001$ \\
\hline IIA & 2.56 & $(2.28,2.87)$ & $<0.001$ & 3.98 & $(3.38,4.69)$ & $<0.001$ \\
\hline IIB & 4.01 & $(3.57,4.49)$ & $<0.001$ & 7.23 & $(6.17,8.49)$ & $<0.001$ \\
\hline IIIA & 5.82 & $(5.24,6.47)$ & $<0.001$ & 10.95 & $(9.42,12.73)$ & $<0.001$ \\
\hline IIIB & 8.42 & $(7.54,9.41)$ & $<0.001$ & 16.36 & $(14.03,19.08)$ & $<0.001$ \\
\hline IIIC & 13.67 & $(12.07,15.47)$ & $<0.001$ & 27.01 & $(22.91,31.85)$ & $<0.001$ \\
\hline IV & 13.07 & $(11.72,14.56)$ & $<0.001$ & 26.32 & $(22.62,30.63)$ & $<0.001$ \\
\hline
\end{tabular}

$H R$ hazard ratio, AJCC American Joint Committee on Cancer

a Adjusted for age, sex, year, race, marital status, tumour grade, radiation, surgery, chemotherapy, number of lymph nodes examined reference, OS, IB, HR 1.63; IIA, HR 2.56; IIB, HR 4.01; IIIA, HR 5.82; IIIB, HR 8.42; IIIC, HR 13.67; IV, HR 13.07, all $P<0.001$; CSS, IB, HR 2.01; IIA, HR 3.98; IIB, HR 7.23; IIIA, HR 10.95; IIIB, HR 16.36; IIIC, HR 27.01; IV, HR 26.32; all $P<0.001$.) Similar results were also observed in the AJCC 7th edition (Supplemental Table 2). The OS and CSS c-indices for the 8th edition were 0.701 and 0.736 , which are largely comparable to those of the AJCC 7th edition (c-indices: 0.701, 0.736). For patients for whom more than 15 lymph nodes were examined, however, OS and CSS c-indices for the AJCC 8th were 0.717 and 0.744, which were higher than those for the AJCC 7th edition system (c-indices: 0.712 and 0.739). For cases in which fewer than 15 lymph nodes were examined, the OS and CSS c-indices were similar as compared between the 7 th and 8 th edition systems (data not shown). These findings suggested that better survival stratification of the new staging system might be independent of the number of lymph nodes examined.

Finally, we investigated stage migration between the 7 th and 8th editions. Supplemental Tables 3 and 4 summarize the main changes and distribution of patients between the two editions. Most modifications occurred in stage III groups relative to their counterparts in the AJCC 7th edition. Overall, downstaging was observed in $12.6 \%$ of cases, whereas $2.3 \%$ of patients were upstaged. Compared with the 7th edition, the percentage of cases in stage IIIA increased (from $11.1 \%$ to $16.9 \%$ ), whereas the proportion of patients in both stage IIIB and IIIC decreased in the 8th edition (IIIB, from $15.1 \%$ to $13.9 \%$; IIIC, from $12.1 \%$ to $7.5 \%)$. As Supplemental Table 4 shows, patients with $\mathrm{T}_{1} \mathrm{~N}_{3 \mathrm{~b}} \mathrm{M}_{0}$ (AJCC 7th IIB) and $\mathrm{T}_{2} \mathrm{~N}_{3 \mathrm{~b}} \mathrm{M}_{0}$ (AJCC 7th IIIA) shifted to advanced-stage IIIB in the AJCC 8th, whereas cases with $\mathrm{T}_{3 \mathrm{~b}} \mathrm{~N}_{3 \mathrm{~b}} \mathrm{M}_{0}$ (AJCC 7th IIIB) shifted to stage IIIC in the AJCC 8th. Patients in AJCC 7th edition stage IIIB $\left(\mathrm{T}_{4 \mathrm{~b}} \mathrm{~N}_{0} \mathrm{M}_{0}, \mathrm{~T}_{4 \mathrm{a}} \mathrm{N}_{2} \mathrm{M}_{0}\right)$ and IIIC $\left(\mathrm{T}_{4 \mathrm{~b}} \mathrm{~N}_{2} \mathrm{M}_{0}, \mathrm{~T}_{4 \mathrm{a}} \mathrm{N}_{3 \mathrm{a}} \mathrm{M}_{0}\right)$ generally dropped to earlier stages in the AJCC 8th edition (IIIA and IIIB). Furthermore, we analyzed survival differences of altered staging groups. As Fig. 4 and Supplemental Fig. 3 show, OS and CSS in patients with $\mathrm{T}_{1} \mathrm{~N}_{3 \mathrm{~b}} \mathrm{M}_{0}$, $\mathrm{T}_{4 \mathrm{~b}} \mathrm{~N}_{0} \mathrm{M}_{0}$, and $\mathrm{T}_{4 \mathrm{~b}} \mathrm{~N}_{2} \mathrm{M}_{0}$ were similar to that in the new staging categories in the AJCC 8th edition, which supported the rationality of the new staging system. However, other revised staging groups were not validated in the current study. Significant survival differences were noted between $\mathrm{T}_{4 \mathrm{a}} \mathrm{N}_{2} \mathrm{M}_{0}$ and stage IIIA (AJCC 8th), which were the opposite of the new stage system. 
a

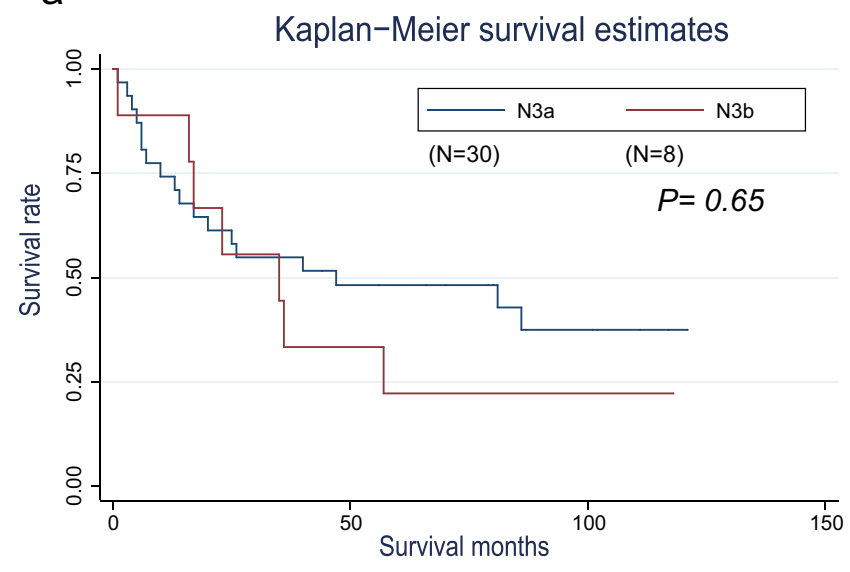

C
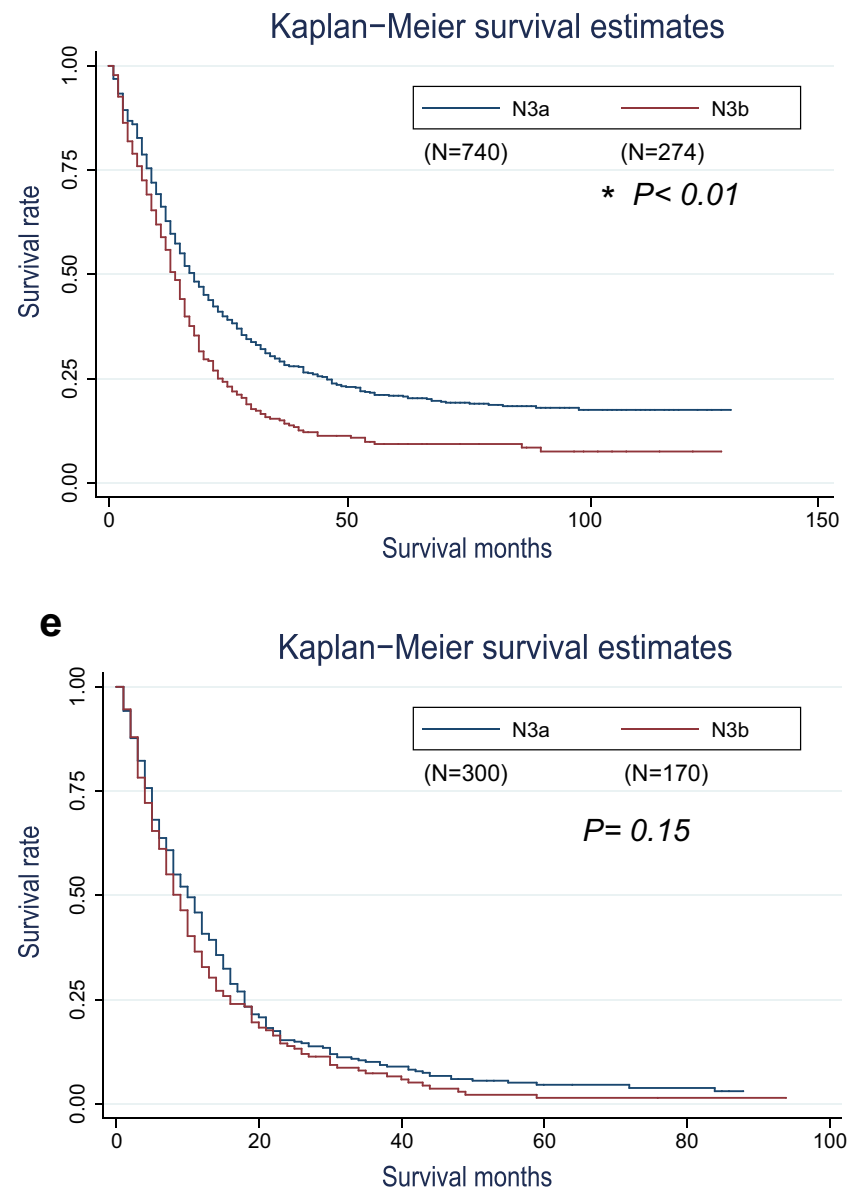

b

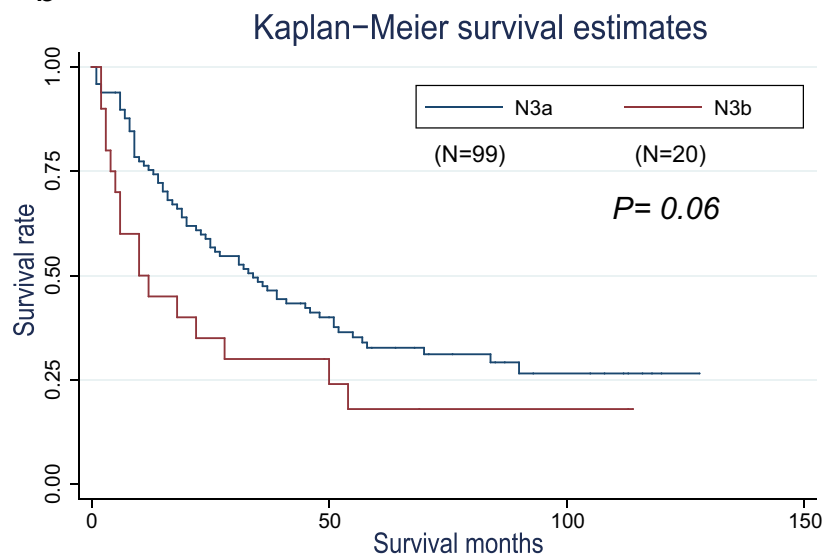

d

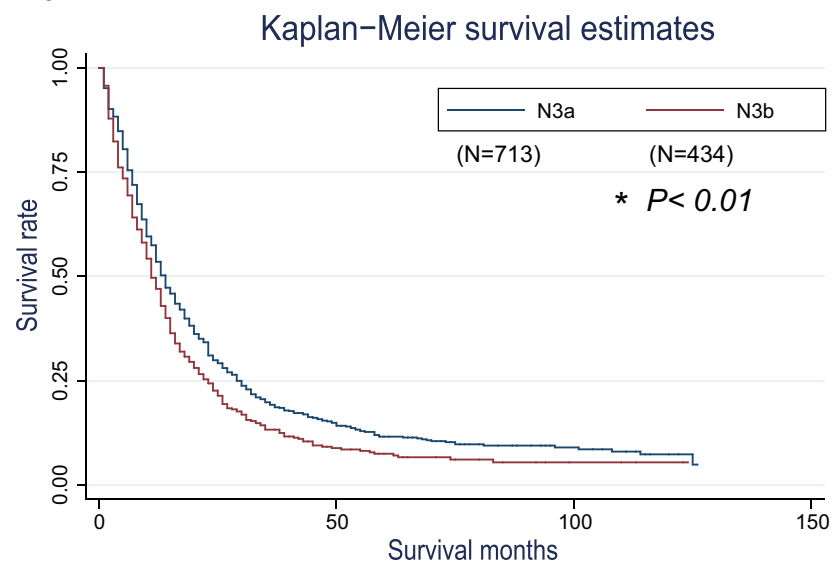

Fig. 2 Comparison of overall survival between N3a and N3b groups stratified by pT category in the AJCC 8th edition. a T1 group. $\mathbf{b}$ T2 group. c T3 group. d T4a group. e T4b group. $P$ was calculated by log-rank test

\section{Discussion}

Based on the population-based cohort of gastric cancer patients from the USA, we observed that the AJCC 8th edition effectively predicted overall and cancer-specific survival outcomes. The principal revision of the new edition was incorporation of $\mathrm{pN} 3 \mathrm{~b}$ as a separate group in the staging system. Lymph node metastasis was considered as an important prognostic factor for gastric cancer [23-26]. Although the optimal number of lymph nodes required for examination remains controversial, it is traditionally recommended that at least 16 lymph nodes should be examined during 


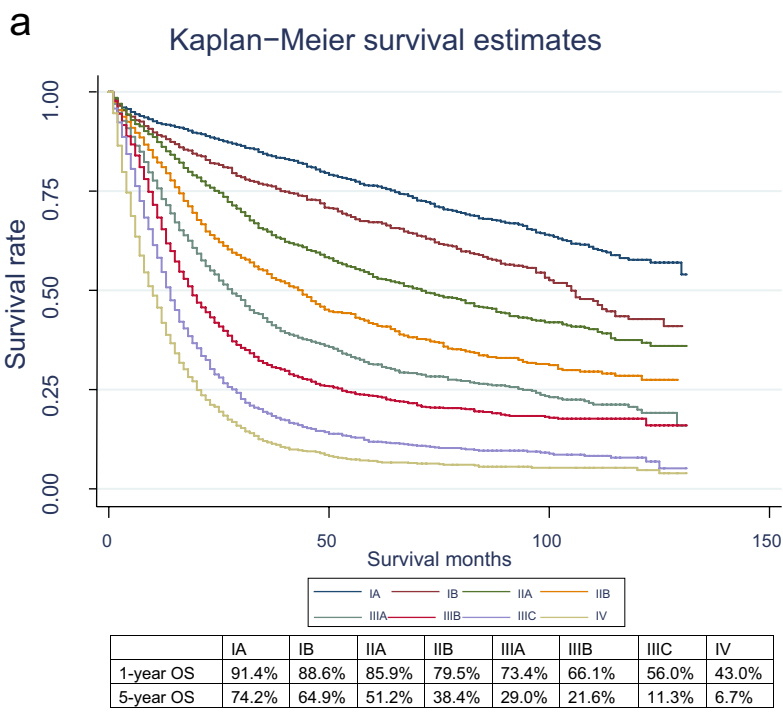

C

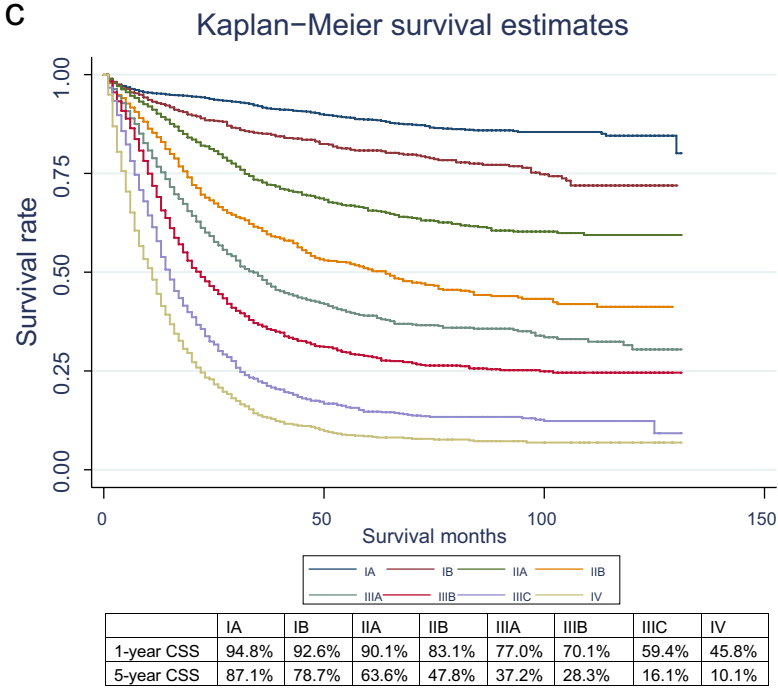

Fig. 3 Kaplan-Meier survival curves of overall and cancer-specific survival according to the AJCC 7th and 8th edition stage system. a Overall survival according to the 7 th edition. b Overall survival

the resection of gastric cancer, especially in Asia [27-30]. Examination of an adequate number of lymph nodes is required for accurate staging, and insufficient lymph node sampling might be associated with a risk of understaging. An increasing number of surgeons in the Western world are also aware of the prognostic significance of the number of lymph nodes removed. Previously, D2 lymphadenectomy was not considered as the standard procedure in Western countries [31, 32], in contrast to the Eastern counterparts. Several randomized clinical trials (RCTs) reported from Western countries demonstrated that D2 lymphadenectomy associated with high perioperative complications and mortality did not provide additional survival benefits [33-35].
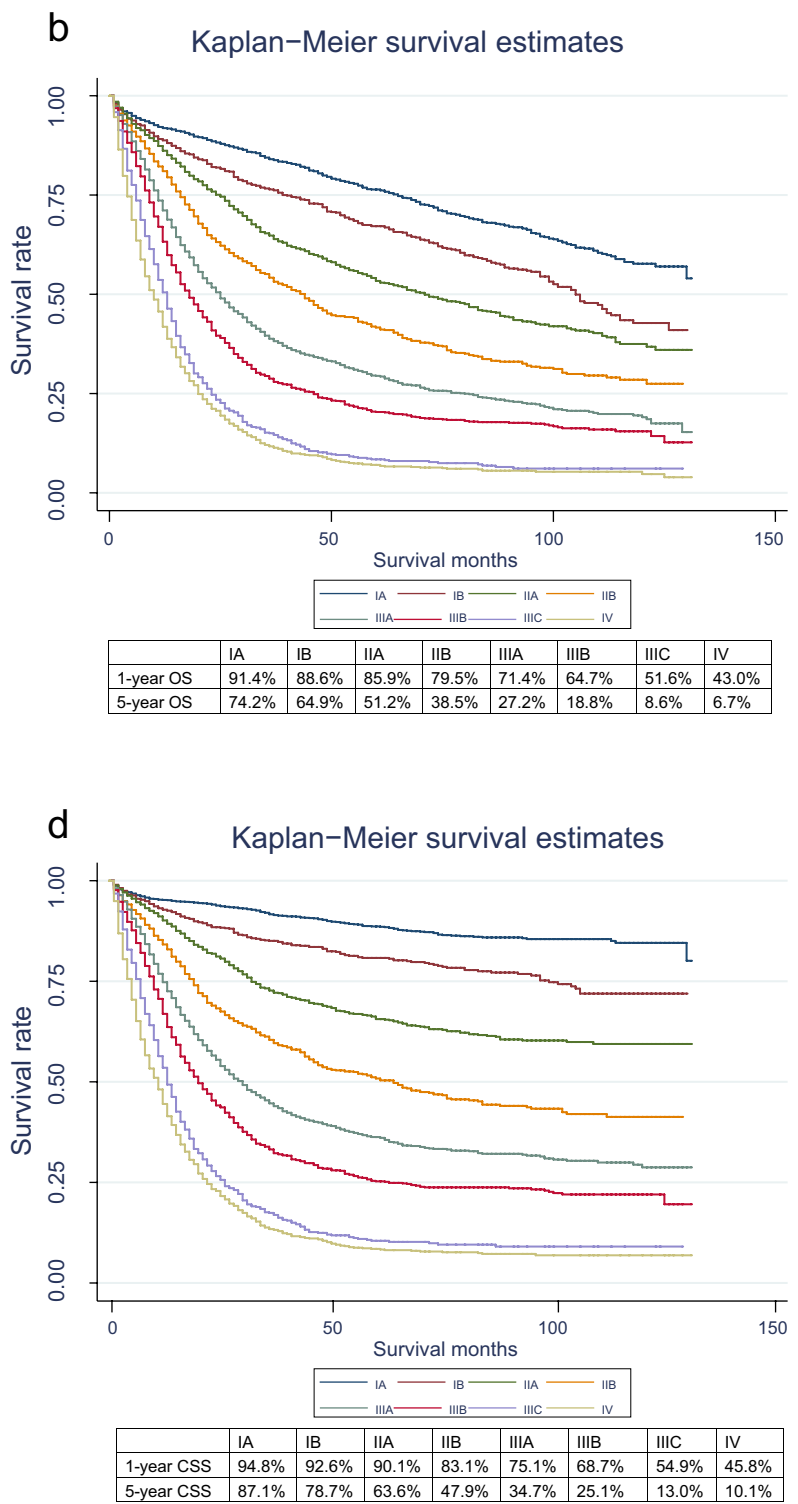

according to the 8th edition. $\mathbf{c}$ Cancer-specific survival according to the 7th edition. $\mathbf{d}$ Cancer-specific survival according to the 8th edition

However, with improved surgical skills and management of complications, the $\mathrm{D} 2$ procedure has been proved to be safe even in Western countries when spleen and pancreas are preserved [36, 37]. Recent RCTs [36, 38] also showed a survival benefit derived from the $\mathrm{D} 2$ procedure. In a recent analysis based on the SEER database, Arsoniadis et al. [39] reported that the percentage of patients with more than 15 lymph nodes examined has been consistently increasing and exceeded 50\% in 2008-2013. It was speculated that adequate lymph node examination might be associated with decreased mortality [39]. Another study from the US Gastric Cancer Collaborative [40] concluded that the number of lymph nodes removed could be considered as an important 
a

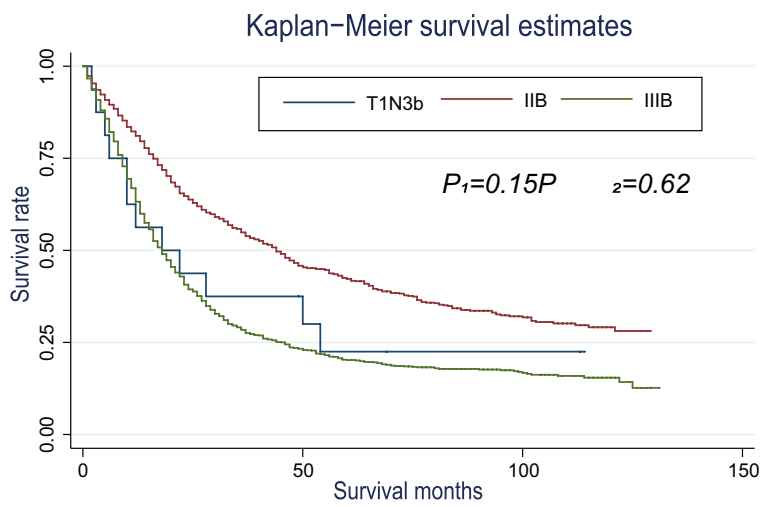

C

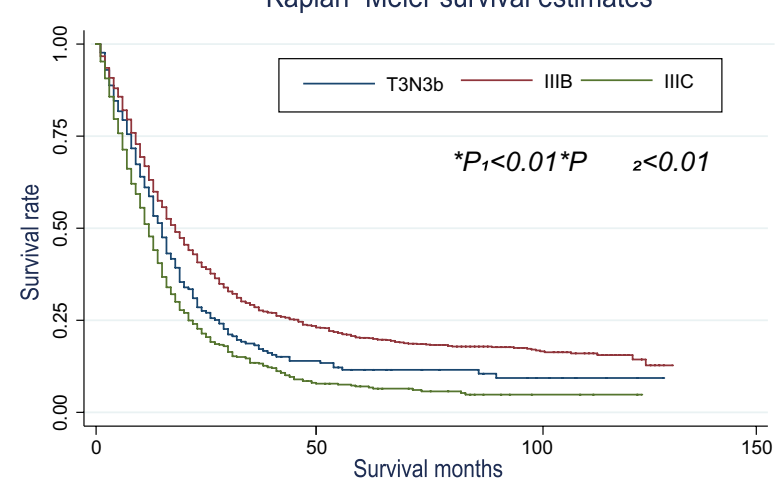

e

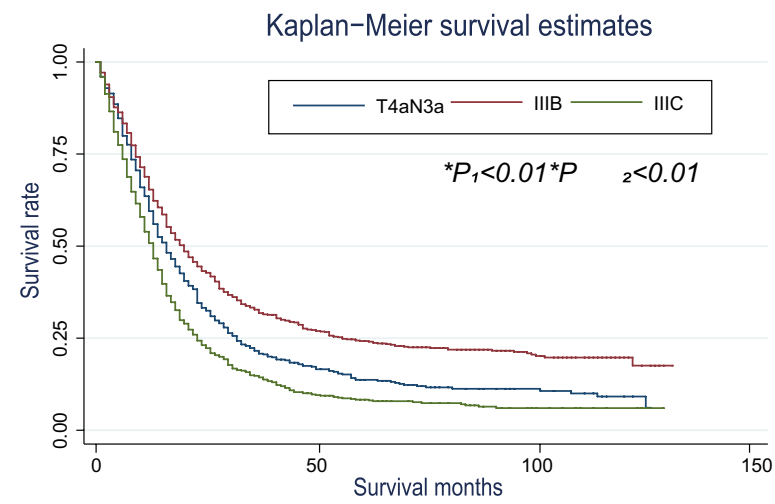

g Kaplan-Meier survival estimates

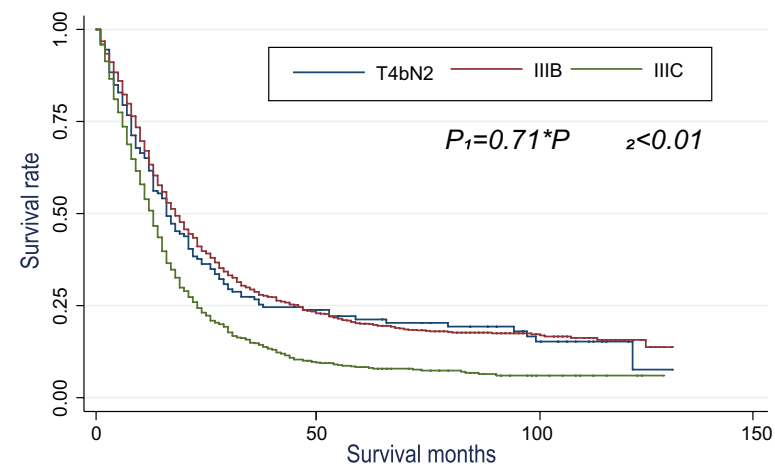

b

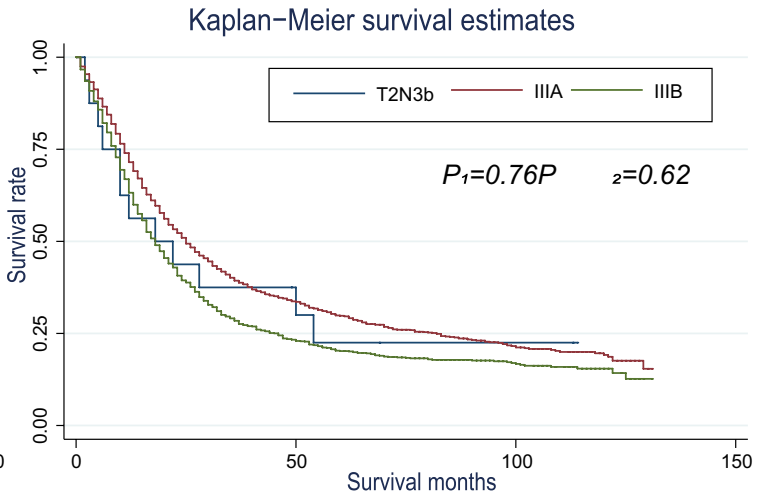

d Kaplan-Meier survival estimates

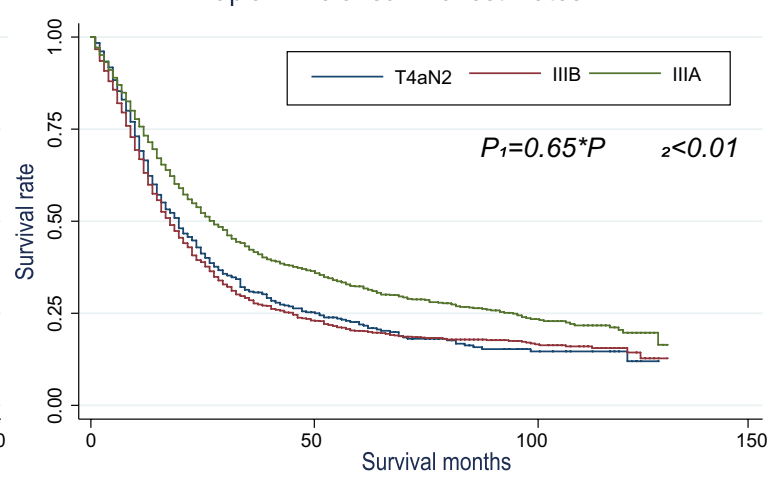

f

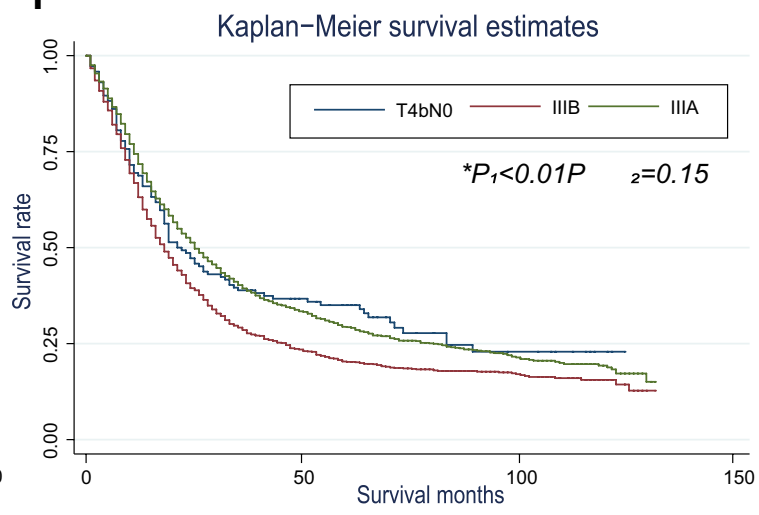


\Fig. 4 Comparison of overall survival among groups with stage migration in the AJCC 8th edition. a T1N3b shifts from IIB to IIIB: $P 1$ for T1N3b and IIB, $P 2$ for T1N3b and IIIB. b T2N3b shifts from IIIA to IIIB: $P 1$ for T2N3b and IIIA, $P 2$ for T2N $3 b$ and IIIB. c T3N3b shifts from IIIB to IIIC: $P 1$ for T3N3b and IIIB, $P 2$ for T3N3b and IIIC. d T4aN2 shifts from IIIB to IIIA: $P 1$ for T4aN2 and IIIB, $P 2$ for T4aN2 and IIIA. e T4aN3a shifts from IIIC to IIIB: $P 1$ for T4aN3a and IIIB, $P 2$ for T4aN3a and IIIC. f T4bN0 shifts from IIIB to IIIA: $P 1$ for T4bN0 and IIIB, $P 2$ for T4bN0 and IIIA. T4bN2 shifts from IIIC to IIIB: $P 1$ for T4bN2 and IIIB, $P 2$ for T4bN2 and IIIC

predictor for long-term prognosis. In keeping with the previous study [16], pN3b was associated with worse survival outcomes in relationship to pN3a in the current study. This trend was even more obvious in patients from whom more than 15 lymph nodes were examined, suggesting that the introduction of $\mathrm{pN} 3 \mathrm{a}$ and $\mathrm{pN} 3 \mathrm{~b}$ as separate groups in staging had significant clinical value.

Compared with the AJCC 7th edition, only stages IA, IB, IIA, and IV remain unchanged in the new edition, with most changes occurring in stage III. The largest subgroup of stage III in the AJCC 8th is IIIA, whereas the largest subgroup in the AJCC 7th was IIIB. In the current study, we validated the staging migration of $\mathrm{T}_{1} \mathrm{~N}_{3} \mathrm{bM}_{0}, \mathrm{~T}_{4 \mathrm{~b}} \mathrm{~N}_{0} \mathrm{M}_{0}$, and $\mathrm{T}_{4 \mathrm{~b}} \mathrm{~N}_{2} \mathrm{M}_{0}$. Justification for other staging changes might require further investigation. Comparable OS and CSS c-indices in the entire cohort indicated that the AJCC 8th performed as well as the AJCC 7th edition stage system in the USA. Importantly, it was noteworthy that the new edition system provided a slight improvement in prognostic discrimination for patients for whom more than 15 lymph nodes were examined, which indicated the significance of examining an adequate number of lymph nodes. The lack of modest improvement in the discriminatory ability of the AJCC 8th relative to the AJCC 7th version in the Western cohort might be the result of examining an insufficient number of lymph nodes.

Several limitations of our study also should be mentioned. First, inherent selection biases were inevitable in the current study. Also, the SEER dataset was unable to provide detailed surgery procedures and postoperative treatment, which might affect the prognosis. Second, we were unable to determine whether patients received neoadjuvant therapy that might affect pathological judgment for staging. Third, for more than half the patients fewer than 15 lymph nodes were examined, which might be associated with the risk of $\mathrm{pN}$ being understaged. When we included those populations, we observed an improvement in prognostic discrimination of the new stage system.

In summary, the AJCC 8th edition stage system refines risk stratification according to the $\mathrm{pN} 3 \mathrm{~b}$ and provides modest to good survival stratification for gastric cancer in the Western cohort as well as does the 7th edition system.
Importantly, an improvement in discriminatory performance of the new edition was observed for cases in which more than 15 lymph nodes were examined.

Acknowledgements We thank the National Cancer Institute's SEER Program for collection of the SEER data.

Author contributions S.L.M., S.J.M., and H.X.K. had the concept for the study; H.X.K., W.W.R., and D.Y.E. conducted data extraction and statistical analysis; H.X.K., L.Z.H., and S.L.M. wrote the first draft of the manuscript; all authors edited and critically revised the final version of the manuscript.

\section{Compliance with ethical standards}

Conflict of interest No potential conflicts of interest to declare.

Funding The work was funded by the Zhejiang provincial medical platform 2015 specialists class B (2015 RCB016); Zhejiang province key science and technology innovation team (2013TD13); Zhejiang Province Medicine Health Key Plan (2014PYA012); and Zhejiang Natural Science Foundation of China (LY18H160019).

Ethical standards Approval from the ethics board was not required.

\section{References}

1. Ferlay J, Shin HR, Bray F, Forman D, Mathers C, Parkin DM. Estimates of worldwide burden of cancer in 2008: GLOBOCAN 2008. Int J Cancer. 2010;127:2893-917.

2. Ferlay J, Parkin DM, Steliarova-Foucher E. Estimates of cancer incidence and mortality in Europe in 2008. Eur J Cancer. 2010;46:765-81.

3. Torre LA, Siegel RL, Ward EM, Jemal A. Global cancer incidence and mortality rates and trends-an update. Cancer Epidemiol Biomark Prev. 2016;25:16-27.

4. Yasuda K, Shiraishi N, Suematsu T, Yamaguchi K, Adachi Y, Kitano S. Rate of detection of lymph node metastasis is correlated with the depth of submucosal invasion in early stage gastric carcinoma. Cancer (Phila). 1999;85:2119-23.

5. Okada K, Fujisaki J, Yoshida T, Ishikawa H, Suganuma T, Kasuga A, et al. Long-term outcomes of endoscopic submucosal dissection for undifferentiated-type early gastric cancer. Endoscopy. 2012;44:122-7.

6. Apicella M, Corso S, Giordano S. Targeted therapies for gastric cancer: failures and hopes from clinical trials. Oncotarget. 2017;8:57654-69.

7. Sobin LH. TNM: principles, history, and relation to other prognostic factors. Cancer (Phila). 2001;91:1589-92.

8. Kim JP, Lee JH, Kim SJ, Yu HJ, Yang HK. Clinicopathologic characteristics and prognostic factors in 10783 patients with gastric cancer. Gastric Cancer. 1998;1:125-33.

9. Warneke VS, Behrens HM, Hartmann JT, Held H, Becker T, Schwarz NT, et al. Cohort study based on the seventh edition of the TNM classification for gastric cancer: proposal of a new staging system. J Clin Oncol. 2011;29:2364-71.

10. Amin MB, Greene FL, Edge SB, Compton CC, Gershenwald JE, Brookland RK, et al. The eighth edition AJCC cancer staging manual: continuing to build a bridge from a population-based to a more "personalized" approach to cancer staging. CA Cancer J Clin. 2017;67:93-9. 
11. Washington K. 7th edition of the AJCC cancer staging manual: stomach. Ann Surg Oncol. 2010;17:3077-9.

12. Sobin LH, Gospodarowicz M, Wittekind C. TNM classification of malignant tumours. Hoboken: Wiley-Blackwell; 2009.

13. Marrelli D, Morgagni P, de Manzoni G, Coniglio A, Marchet A, Saragoni L, et al. Prognostic value of the 7th AJCC/UICC TNM classification of noncardia gastric cancer: analysis of a large series from specialized Western centers. Ann Surg. 2012;255:486-91.

14. Wang W, Sun XW, Li CF, Lv L, Li YF, Chen YB, et al. Comparison of the 6th and 7th editions of the UICC TNM staging system for gastric cancer: results of a Chinese single-institution study of 1,503 patients. Ann Surg Oncol. 2011;18:1060-7.

15. Ahn HS, Lee HJ, Hahn S, Kim WH, Lee KU, Sano T, et al. Evaluation of the seventh American Joint Committee on Cancer/International Union Against Cancer Classification of gastric adenocarcinoma in comparison with the sixth classification. Cancer (Phila). 2010;116:5592-8.

16. Sano T, Coit DG, Kim HH, Roviello F, Kassab P, Wittekind C, et al. Proposal of a new stage grouping of gastric cancer for TNM classification: international gastric cancer association staging project. Gastric Cancer. 2017;20:217-25.

17. Amin MB, Edge S, Greene F, et al. AJCC cancer staging manual. 8th ed. Cham: Springer International; 2016.

18. Markar SR, Karthikesalingam A, Jackson D, Hanna GB. Long-term survival after gastrectomy for cancer in randomized, controlled oncological trials: comparison between West and East. Ann Surg Oncol. 2013;20:2328-38.

19. Strong VE, Song KY, Park CH, Jacks LM, Gonen M, Shah M, et al. Comparison of gastric cancer survival following R0 resection in the United States and Korea using an internationally validated nomogram. Ann Surg. 2010;251:640-6.

20. Therneau TM, Grambsch PMG. Modeling survival data: extending the Cox model. New York: Springer-Verlag; 2000.

21. Harrell FE Jr, Lee KL, Mark DB. Multivariable prognostic models: issues in developing models, evaluating assumptions and adequacy, and measuring and reducing errors. Stat Med. 1996;15:361-87.

22. Wang T, Hua D, Yu H. Prognostic significance of the number of negative lymph nodes in postoperative locally advanced gastric cancer patients. Clin Oncol Cancer Res. 2009;6:418-21.

23. D’Ugo D, Pacelli F, Persiani R, Pende V, Ianni A, Papa V, et al. Impact of the latest TNM classification for gastric cancer: retrospective analysis on 94 D2 gastrectomies. World J Surg. 2002;26:672-7.

24. Del Rio P, Dell'Abate P, Soliani P, Arcuri MF, Tacci S, Ziegler S, et al. Old and new TNM in carcinoma of the gastric antrum: analysis of our personal experience. J Gastrointest Surg. 2003;7:912-6.

25. Chae S, Lee A, Lee JH. The effectiveness of the new (7th) UICC N classification in the prognosis evaluation of gastric cancer patients: a comparative study between the 5th/6th and 7th UICC N classification. Gastric Cancer. 2011;14:166-71.

26. Zhou Y, Zhang GJ, Wang J, Zheng KY, Fu W. Current status of lymph node micrometastasis in gastric cancer. Oncotarget. 2017;8:51963-9.
27. Maruyama K, Okabayashi K, Kinoshita T. Progress in gastric cancer surgery in Japan and its limits of radicality. World J Surg. 1987;11:418-25.

28. Katai H, Yoshimura K, Maruyama K, Sasako M, Sano T. Evaluation of the New International Union against Cancer TNM staging for gastric carcinoma. Cancer (Phila). 2000;88:1796-800.

29. Japanese Gastric Cancer Association. Japanese classification of gastric carcinoma: 3rd English edition. Gastric Cancer. 2011;14:101-12.

30. Lee HK, Yang HK, Kim WH, Lee KU, Choe KJ, Kim JP. Influence of the number of lymph nodes examined on staging of gastric cancer. Br J Surg. 2001;88:1408-12.

31. Degiuli M, De Manzoni G, Di Leo A, D’Ugo D, Galasso E, Marrelli D, et al. Gastric cancer: current status of lymph node dissection. World J Gastroenterol. 2016;22:2875-93.

32. Giuliani A, Miccini M, Basso L. Extent of lymphadenectomy and perioperative therapies: two open issues in gastric cancer. World $\mathbf{J}$ Gastroenterol. 2014;20:3889-904.

33. Wong J, Jackson P. Gastric cancer surgery: an American perspective on the current options and standards. Curr Treat Options Oncol. 2011;12:72-84.

34. Bonenkamp JJ, Hermans J, Sasako M, van de Velde CJ, Welvaart K, Songun I, et al. Extended lymph-node dissection for gastric cancer. N Engl J Med. 1999;340:908-14.

35. Cuschieri A, Fayers P, Fielding J, Craven J, Bancewicz J, Joypaul V, et al. Postoperative morbidity and mortality after D1 and D2 resections for gastric cancer: preliminary results of the MRC randomised controlled surgical trial. The Surgical Cooperative Group. Lancet. 1996;347:995-9.

36. Degiuli M, Sasako M, Ponti A, Italian Gastric Cancer Study G. Morbidity and mortality in the Italian Gastric Cancer Study Group randomized clinical trial of D1 versus D2 resection for gastric cancer. Br J Surg. 2010;97:643-9.

37. Degiuli M, Sasako M, Calgaro M, Garino M, Rebecchi F, Mineccia M, et al. Morbidity and mortality after D1 and D2 gastrectomy for cancer: interim analysis of the Italian Gastric Cancer Study Group (IGCSG) randomised surgical trial. Eur J Surg Oncol. 2004;30:303-8.

38. Songun I, Putter H, Kranenbarg EM, Sasako M, van de Velde CJ. Surgical treatment of gastric cancer: 15 -year follow-up results of the randomised nationwide Dutch D1D2 trial. Lancet Oncol. 2010;11:439-49.

39. Arsoniadis EG, Marmor S, Diep GK, Hui JYC, Jensen EH, Tuttle TM. Survival rates for patients with resected gastric adenocarcinoma finally have increased in the United States. Ann Surg Oncol. 2017;24:3361-7.

40. Gholami S, Janson L, Worhunsky DJ, Tran TB, Squires MH 3rd, Jin LX, et al. Number of lymph nodes removed and survival after gastric cancer resection: an analysis from the US Gastric Cancer Collaborative. J Am Coll Surg. 2015;221:291-9. 